\title{
A Spectral Approach for Time-Fractional Diffusion and Subdiffusion Equations in a Large Interval
}

\section{Haniye Dehestani ${ }^{a}$, Yadollah Ordokhani ${ }^{a}$ and Mohsen Razzaghi ${ }^{b}$}

\author{
${ }^{a}$ Department of Mathematics, Faculty of Mathematical Sciences, Alzahra \\ University \\ 1993893973 Tehran, Iran \\ ${ }^{b}$ Department of Mathematics and Statistics, Mississippi State University \\ MS 39762 Starkville, USA \\ E-mail(corresp.): ordokhani@alzahra.ac.ir \\ E-mail: h.dehestani@alzahra.ac.ir \\ E-mail: razzaghi@math.msstate.edu
}

Received September 21, 2020; revised October 25, 2021; accepted October 25, 2021

\begin{abstract}
In this paper, we concentrate on a class of time-fractional diffusion and subdiffusion equations. To solve the mentioned problems, we construct twodimensional Genocchi-fractional Laguerre functions (G-FLFs). Then, the pseudooperational matrices are used to convert the proposed equations to systems of algebraic equations. The properties of pseudo-operational matrices have reflected well in the process of the numerical technique and create an approximate solution with high precision. Finally, several examples are presented to illustrate the accuracy and effectiveness of the technique.
\end{abstract}

Keywords: Genocchi-fractional Laguerre functions, collocation method, time-fractional diffusion equations, time-fractional subdiffusion equations, pseudo-operational matrix.

AMS Subject Classification: 65M70; 35R11; 65M15.

\section{Introduction}

Recently, the fractional calculus has been applied to various branches of science such as signal processing, fluid mechanics, diffusion processes, and continuum, etc. $[1,15,30,35]$. 
In recent years, fractional partial differential equations (FPDEs) have attracted considerable attention because of their application in the modeling of various phenomena. The fractional diffusion equation is an important type of FPDEs which has been studied widely. The physical application of fractional diffusion equations (FDEs) is related to the phenomena of anomalous diffusion in transport processes through complex and/or disordered systems including fractal media and fractional kinetic equations have proved particularly useful in the context of anomalous slow diffusion [32].

Moreover, the time-fractional subdiffusion equations (FSDEs) are created by replacing the time derivative in ordinary diffusion by a fractional derivative of order $0<\gamma<1$. This class of equation is achieved from anomalous diffusive systems. The FSDEs are considered as the following form $[7,17,36]$ :

$$
\frac{\partial u}{\partial t}=D_{t}^{1-\gamma}\left(\kappa_{\gamma} \frac{\partial^{2} u}{\partial x^{2}}\right)+f(x, t), \quad 0<\gamma \leq 1
$$

where $\kappa_{\gamma}$ generalized diffusion constant, $\gamma$ is the anomalous diffusion exponent and $D_{t}^{\gamma}$ is the $\gamma$ th-order Caputo derivative operator [46].

Since analytic techniques for finding the solutions of FDEs and FSDEs cannot work well. Therefore, the researchers seek to establish efficient and reliable numerical techniques. Note that various numerical techniques have existed to solve these problems that we are presenting some of them. Mustapha [34] solved sub-diffusion equations by an implicit finite-difference time-stepping method, Jiang et al. [23] derived and utilized high-order finite element methods for timefractional partial differential equations, Si et al. [40] presented Haar wavelet operational matrix for solving fractional partial differential equations with time-space variable coefficients, Gao et al. [17] have discussed on the fractional sub-diffusion equations with the help of the compact finite difference scheme, Wang et al. [43] investigated the solution of the modified anomalous fractional sub-diffusion equation and the fractional diffusion-wave equation by compact difference schemes, Yaseen et al. [44] derived a cubic trigonometric B-spline collocation approach for the fractional sub-diffusion equations, Dehestani et al. [8] established the fractional-order Legendre-Laguerre functions for approximate solution of fractional partial differential equations. For more details about the proposed equation, the reader is advised to see $[4,11,13,18,20,24,25,45]$.

Many references on the spectral approach for solving different kinds of fractional partial differential equations have been presented. Researchers have also come up with successful results with this method. For example, fractional-order Jacobi pseudo-spectral method [6], operational method based on hybrid Legendre functions [33], Chebyshev wavelets collocation method [48], RBF method and Chebyshev polynomials [26], Haar wavelet collocation method [3], wavelet collocation method [41] etc., (see [2,8,27,37,39] and the references therein).

The main purpose of the present numerical scheme is solving FDEs and FSDEs based on the G-FLFs and collocation method. First, by combining the Genocchi polynomials (GPs) $[21,22,29]$ and fractional-order Laguerre functions (FLFs), we introduce the bivariate G-FLFs. Then, by means of pseudooperational matrices (POMs), FDEs and FSDEs are reduced to a system of algebraic equations. 
In this work, we apply FLFs to approximate the time variable because the behavior of the physical problems for large time is important. The Laguerre polynomials are orthogonal in the semi-infinite interval and also these polynomials are appropriate for approximating problems in semi-infinite intervals. Also, we utilize GPs for approximating variable $x$ because these polynomials are superior to other polynomials. So that the advantages of these polynomials are mentioned in $[9,10]$.

The paper is structured as follows: In the next section, we present the framework of numerical technique, which contains the GPs, FLFs and G-FLFs. Pseudo-operational matrices of integration, derivative and some necessary formulas of G-FLFs are introduced in Section 3. In Section 4, the formulation of the method based on G-FLFs is explained. In Section 5, we discuss error estimation. To verify the accuracy and efficiency of the proposed approach, we examine several test problems in Section 6. Finally, the conclusions of the proposed numerical scheme are discussed in last section.

\section{Combination of GPs and FLFs}

This section introduces the Genocchi-fractional Laguerre functions and their features.

\subsection{Genocchi polynomials}

The Genocchi polynomials of degree $m$, which is defined as follows $[9,10]$ :

$$
G_{m}(x)=\sum_{k=0}^{m}\left(\begin{array}{l}
m \\
k
\end{array}\right) g_{m-k} x^{k}
$$

where $g_{k}$ denotes the Genocchi numbers. Now, we mention some significant properties of Genocchi polynomials [12]:

$$
\int_{a}^{b} G_{m}(x) d x=\frac{G_{m+1}(b)-G_{m+1}(a)}{m+1}, \quad \int_{0}^{x} G_{m}(t) d t=\frac{G_{m+1}(x)-g_{m+1}}{m+1} .
$$

Any function $g$ defined over the interval $[0,1]$ can be expanded in terms of GPs which is described in detail in [12].

\subsection{Fractional-order Laguerre functions}

The analytic form of FLFs is given by [8]:

$$
L_{n}^{\beta}(t)=\sum_{k=0}^{n} \frac{(-1)^{k} n !}{(k !)^{2}(n-k) !} t^{\beta k} .
$$

The orthogonal property of the FLFs with respect to the weight function $w_{\beta}(t)=t^{\beta-1} \exp \left(-t^{\beta}\right)$ are presented in [8], which the following formula is gotten:

$$
\int_{0}^{\infty} w_{\beta}(t) L_{m}^{\beta}(t) L_{n}^{\beta}(t) d t=\frac{1}{\beta} \delta_{m n}
$$


where $\delta_{m n}$ is the Kronecker symbol. In addition, any function $h$ defined over the interval $[0, \infty)$, can be approximated by FLFs which is described in detail in $[8]$.

\subsection{Genocchi-fractional Laguerre functions}

The G-FLFs $\mathbf{G L}_{m n}^{\beta}(x, t)$ are defined on the interval $\Omega=(x, t) \in[0,1] \times[0, \infty)$, as

$$
\mathbf{G L}_{m n}^{\beta}(x, t)=G_{m}(x) L_{n}^{\beta}(t)=\sum_{i=0}^{m} \sum_{j=0}^{n}\left(\begin{array}{c}
m \\
i
\end{array}\right) \frac{(-1)^{j} n !}{(j !)^{2}(n-j) !} g_{m-i} x^{i} t^{\beta j}
$$

$m=1,2, \ldots, M, n=0,1, \ldots, N$. Any arbitrary function $f(x, t) \in L^{2}(\Omega)$ can be expanded with the first $M(N+1)$-terms of G-FLFs as

$$
f(x, t)=\sum_{m=1}^{M} \sum_{n=0}^{N} f_{m n} \mathbf{G L}_{m n}^{\beta}(x, t) \simeq F^{T} \mathbf{G L}^{\beta}(x, t),
$$

where

$$
\begin{gathered}
\mathbf{G L}^{\beta}(x, t)=\left[\mathbf{G L}_{m n}^{\beta}(x, t)\right]^{T}=\left[\mathbf{G L}_{m}^{\beta}(x, t)\right]^{T}, \quad F=\left[f_{m n}\right]^{T}, \\
m=1,2, \ldots, M, \quad n=0,1, \ldots, N .
\end{gathered}
$$

\section{POMs and some necessary formulas}

The present section is devoted to the technique of computing the POMs.

\subsection{POM of integration with respect to $x$}

The integration of the G-FLFs, $\mathbf{G L}^{\beta}(x, t)$ with respect to the variable $x$ is determined by the following relation:

$$
\int_{0}^{x} \xi^{r} \mathbf{G} \mathbf{L}^{\beta}(\xi, t) d \xi \simeq x^{r+1} P_{r} \mathbf{G L}^{\beta}(x, t), \quad r=0,1,2, \ldots,
$$

where $P_{r}$ denotes $M(N+1) \times M(N+1)$ POM of integration with respect to $x$. To introduce the proposed matrix, we introduce the POM of GPs as follows:

$$
\int_{0}^{x} \xi^{r} G(\xi) d \xi \simeq x^{r+1} \bar{P}_{r} G(x) .
$$

Now, to compute each element of the POM for GPs, we follow the below process:

$$
\int_{0}^{x} \xi^{r} G_{m}(\xi) d \xi=\int_{0}^{x}\left(\sum_{k=0}^{m}\left(\begin{array}{c}
m \\
k
\end{array}\right) g_{m-k} \xi^{k+r}\right) d \xi=x^{r+1} \sum_{k=0}^{m}\left(\begin{array}{c}
m \\
k
\end{array}\right) \frac{g_{m-k}}{k+r+1} x^{k} .
$$


Also, it can be written

$$
x^{k} \simeq \sum_{i=1}^{M} b_{k i} G_{i}(x) .
$$

Then, by substituting the aforesaid equation in previous equation, we achieve

$$
\begin{aligned}
& \int_{0}^{x} \xi^{r} G_{m}(\xi) d \xi \simeq x^{r+1} \sum_{k=0}^{m}\left(\begin{array}{c}
m \\
k
\end{array}\right) \frac{g_{m-k}}{k+r+1}\left[\sum_{i=1}^{M} b_{k i} G_{i}(x)\right] \\
& =x^{r+1} \sum_{i=1}^{M}\left(\sum_{k=0}^{m} \kappa_{m k i}\right) G_{i}(x)=x^{r+1}\left[\sum_{k=0}^{m} \kappa_{m k 1}, \sum_{k=0}^{m} \kappa_{m k 2}, \ldots, \sum_{k=0}^{m} \kappa_{m k M}\right] G(x),
\end{aligned}
$$

where $\kappa_{m k i}=\left(\begin{array}{c}m \\ k\end{array}\right) \frac{g_{m-k}}{k+r+1} b_{k i}$. Therefore, we obtain the integral POM of G-FLFs as

$$
P_{r}=\bar{P}_{r} \otimes I_{(N+1) \times(N+1)}=\left[\bar{P}_{i j}^{r} I_{(N+1) \times(N+1)}\right]_{M(N+1) \times M(N+1)}, i, j=1,2, \ldots, M .
$$

\subsection{POM of fractional integration with respect to $t$}

In this part, we present the pseudo-operational matrix of fractional integration of order $\nu>0$ for G-FLFs vector $\mathbf{G L}^{\beta}(x, t)$ with respect to $t$ as

$$
I_{t}^{\nu}\left[t^{l} \mathbf{G L}^{\beta}(x, t)\right] \simeq t^{\nu+l} \Upsilon_{l}^{\beta, \nu} \mathbf{G L}^{\beta}(x, t), \quad l=0,1,2, \ldots
$$

Here $\Upsilon_{l}^{\beta, \nu}$ denotes the pseudo-operational matrix of fractional integration of order $\nu$ and $\Upsilon_{l}^{\beta, \nu}=I \otimes \Theta_{l}^{\beta, \nu}$. So that

$$
I_{t}^{\nu}\left[t^{l} L^{\beta}(t)\right]=t^{\nu+l} \Theta_{l}^{\beta, \nu} L^{\beta}(t)
$$

and $\Theta_{l}^{\beta, \nu}$ is the fractional integral pseudo-operational matrix of FLFs, which is defined in [8].

\subsection{POM of fractional derivative with respect to $t$}

In the current section, we consider the pseudo-operational matrix of the fractional derivative of order $\nu>0$ with respect to $t$. Then, we have

$$
D_{t}^{\nu}\left[t^{s} \mathbf{G L}^{\beta}(x, t)\right] \simeq t^{s-\nu} \Phi_{s}^{\beta, \nu} \mathbf{G L}^{\beta}(x, t), \quad s=0,1,2, \ldots
$$

Here $\Phi_{s}^{\beta, \nu}$ denotes the pseudo-operational matrix of fractional derivative for G-FLFs. To get the desired goal, we obtain the pseudo-operational matrix of the fractional derivative for FLFs as

$$
D_{t}^{\nu}\left[t^{s} L^{\beta}(t)\right] \simeq t^{s-\nu} Q_{s}^{\beta, \nu} L^{\beta}(t)
$$


Each component of the POM is determined by the following relations:

$$
\begin{aligned}
D_{t}^{\nu}\left[t^{s} L^{\beta}(t)\right] & =D_{t}^{\nu}\left(\sum_{k=0}^{n} \frac{(-1)^{k} n !}{(k !)^{2}(n-k) !} t^{\beta k+s}\right) \\
& =t^{s-\nu} \sum_{k=\left\lceil\frac{s-\nu}{\beta}\right\rceil}^{n} \frac{(-1)^{k} n !}{(k !)^{2}(n-k) !} \frac{\Gamma(\beta t+s+1)}{\Gamma(\beta t+s-\nu+1)} t^{\beta k}
\end{aligned}
$$

Also, it can be written $t^{\beta k} \simeq \sum_{i=0}^{N} c_{k i}^{\beta} L_{i}^{\beta}(t)$. From Equation (3.3), we get

$$
D_{t}^{\nu}\left[t^{s} L^{\beta}(t)\right] \simeq t^{s-\nu} \sum_{i=0}^{N}\left(\sum_{k=\left\lceil\frac{s-\nu}{\beta}\right\rceil}^{n} \lambda_{n k i}\right) L_{i}^{\beta}(t),
$$

where $\lambda_{n k i}=\frac{(-1)^{k} n !}{(k !)^{2}(n-k) !} \frac{\Gamma(\beta t+s+1)}{\Gamma(\beta t+s-\nu+1)} c_{k i}^{\beta}$. Consequently, the POM of the fractional derivative for G-FLFs is obtained by using the pseudo-operational matrix of the fractional derivative of FLFs as $\Phi_{s}^{\beta, \nu}=I \otimes Q_{s}^{\beta, \nu}$.

\subsection{Necessary relations}

In the proceeding of the numerical algorithm, we need to calculate the following formula:

$$
\int_{0}^{1} x \mathbf{G L}^{\beta}(x, t) d x=\Lambda L^{\beta}(t) .
$$

To achieve the sought goal, we use the below relation

$$
\mathbf{G L}^{\beta}(x, t)=\Psi(x) L^{\beta}(t),
$$

where

$$
\Psi(x)=\operatorname{diag}[G(x), G(x), \ldots, G(x)]_{M(N+1) \times(N+1)} .
$$

Thus, the matrix $\Lambda$ is obtained as

$$
\Lambda=\operatorname{diag}[S, S, \ldots, S]_{M(N+1) \times(N+1)} .
$$

According to the Genocchi polynomials properties, we get the vector $S$ as

$$
S=\int_{0}^{1} x G(x) d x=\left[\begin{array}{llll}
\frac{G_{2}(1)}{2}-\frac{G_{3}(1)}{6} & \frac{G_{3}(1)}{3}-\frac{G_{4}(1)}{12} & \ldots & \frac{G_{M+1}(1)}{M+1}-\frac{G_{M+2}(1)}{(M+1)(M+2)}
\end{array}\right]^{T} .
$$

\section{Construction of the numerical method}

This article is devoted to investigating the numerical solution of the following form of the time-fractional diffusion equation:

$$
D_{t}^{\nu} u(x, t)=F\left(u, \frac{\partial u}{\partial x}, \frac{\partial u}{\partial t}, \frac{\partial^{2} u}{\partial x \partial t}, \frac{\partial^{3} u}{\partial x^{2} \partial t}\right), \quad 0<\nu \leq 2
$$


and the time-fractional subdiffusion equation

$$
\frac{\partial u}{\partial t}=D_{t}^{1-\gamma}\left(\frac{\partial^{2} u}{\partial x^{2}}\right)+F\left(x, t, u, \frac{\partial u}{\partial x}, \frac{\partial^{2} u}{\partial x^{2}}\right), \quad 0<\gamma \leq 1,
$$

subject to the initial conditions $u(x, 0)=\omega_{0}(x), \frac{\partial u(x, 0)}{\partial t}=\omega_{1}(x), x \in[0,1]$, and boundary conditions $u(0, t)=\varphi_{0}(t), u(1, t)=\varphi_{1}(t), t \in[0, \infty)$, where $F$ is a nonlinear function and the functions $\omega_{0}(x), \omega_{1}(x), \varphi_{0}(t)$ and $\varphi_{1}(t)$ are known in the domain $\Omega$, and also $u$ is an unknown function to be calculated. The involved fractional derivatives are described in the Caputo sense. In the present approach, we approximate the higher order derivative by G-FLFs as

$$
\frac{\partial^{4} u(x, t)}{\partial x^{2} \partial t^{2}} \simeq U^{T} \mathbf{G L}^{\beta}(x, t) .
$$

By integrating from Equation (4.3) with respect to $t$ and utilizing the initial conditions and the POM of integration introduced in Section 3.2, we obtain

$$
\frac{\partial^{3} u(x, t)}{\partial x^{2} \partial t} \simeq t U^{T} \Upsilon_{0}^{\beta, 1} \mathbf{G L}^{\beta}(x, t)+\omega_{1}^{\prime \prime}(x),
$$

integrating from Equation (4.4), to obtain

$$
\frac{\partial^{2} u(x, t)}{\partial x^{2}} \simeq t^{2} U^{T} \Upsilon_{0}^{\beta, 1} \Upsilon_{1}^{\beta, 1} \mathbf{G L}^{\beta}(x, t)+t \omega_{1}^{\prime \prime}(x)+\omega_{0}^{\prime \prime}(x) .
$$

In the following, by integrating Equation (4.5) with respect to $x$ and using the POM of integration in Equation (3.1), we have

$$
\begin{aligned}
\frac{\partial}{\partial x} u(x, t) \simeq & x t^{2} U^{T} \Upsilon_{0}^{\beta, 1} \Upsilon_{1}^{\beta, 1} P_{0} \mathbf{G L}^{\beta}(x, t)+t\left(\omega_{1}^{\prime}(x)-\omega_{1}^{\prime}(0)\right) \\
& +\left(\omega_{0}^{\prime}(x)-\omega_{0}^{\prime}(0)\right)+\frac{\partial u(0, t)}{\partial x}
\end{aligned}
$$

and

$$
\begin{aligned}
u(x, t) \simeq & x^{2} t^{2} U^{T} \Upsilon_{0}^{\beta, 1} \Upsilon_{1}^{\beta, 1} P_{0} P_{1} \mathbf{G L}^{\beta}(x, t)+t\left(\omega_{1}(x)-\omega_{1}(0)-x \omega_{1}^{\prime}(0)\right) \\
& +\left(\omega_{0}(x)-\omega_{0}(0)-x \omega_{0}^{\prime}(0)\right)+x \frac{\partial u(0, t)}{\partial x}+u(0, t)
\end{aligned}
$$

To achieve the approximate solution, it is necessary to determine unknown function $\frac{\partial u(0, t)}{\partial x}$. Thus, integrate Equation (4.6) with respect to $x$ from 0 to 1 as

$$
\begin{aligned}
\frac{\partial u(0, t)}{\partial x} \simeq & u(1, t)-u(0, t)-t^{2} U^{T} \Upsilon_{0}^{\beta, 1} \Upsilon_{1}^{\beta, 1} P_{0} \Lambda L^{\beta}(t) \\
& +t\left(\omega_{1}(1)-\omega_{1}(0)-\omega_{1}^{\prime}(0)\right)-\left(\omega_{0}(1)-\omega_{0}(0)-\omega_{0}^{\prime}(0)\right)
\end{aligned}
$$

Then, by substituting the above relation in Equations (4.6) and (4.7), the desired results are obtained. In a similar way, in order to express the approximation of the other unknown functions, we take integration from Equation (4.3) and follow the process below:

$$
\frac{\partial^{3} u(x, t)}{\partial x \partial t^{2}} \simeq x U^{T} P_{0} \mathbf{G L}^{\beta}(x, t)+\frac{\partial^{3} u(0, t)}{\partial x \partial t^{2}}=\frac{\partial^{3} u_{M N}(x, t)}{\partial x \partial t^{2}},
$$


and also,

$$
\frac{\partial^{2} u(x, t)}{\partial t^{2}} \simeq x^{2} U^{T} P_{0} P_{1} \mathbf{G L}^{\beta}(x, t)+x \frac{\partial^{3} u(0, t)}{\partial x \partial t^{2}}+\varphi_{0}^{\prime \prime}(t)=\frac{\partial^{2} u_{M N}(x, t)}{\partial t^{2}} .
$$

In order to calculate the unknown statement $\frac{\partial^{3} u(0, t)}{\partial x \partial t^{2}}$, integrating Equation (4.8) from 0 to 1 as

$$
\frac{\partial^{3} u(0, t)}{\partial x \partial t^{2}} \simeq \varphi_{1}^{\prime \prime}(t)-\varphi_{0}^{\prime \prime}(t)-U^{T} P_{0} \Lambda L^{\beta}(t)
$$

Then, from Equations (4.9) and (4.10), we get

$$
\begin{aligned}
\frac{\partial u(x, t)}{\partial t} \simeq & x^{2} t U^{T} P_{0} P_{1} \Upsilon_{0}^{\beta, 1} \mathbf{G L}^{\beta}(x, t)+x\left(\varphi_{1}^{\prime}(t)-\varphi_{1}^{\prime}(0)-\varphi_{0}^{\prime}(t)+\varphi_{0}^{\prime}(0)\right. \\
& \left.-t U^{T} P_{0} \Lambda \Theta_{0}^{\beta, 1} L^{\beta}(t)\right)+\varphi_{0}^{\prime}(t)-\varphi_{0}^{\prime}(0)+\omega_{1}(x)
\end{aligned}
$$

By applying the properties of Caputo fractional derivative operator of order $0<\nu \leq 1$ in Equation (4.11), we have

$$
\begin{aligned}
& D_{t}^{\nu} u(x, t)=I_{t}^{1-\nu}\left(\frac{\partial u(x, t)}{\partial t}\right) \simeq x^{2} t^{2-\nu} U^{T} P_{0} P_{1} \Upsilon_{0}^{\beta, 1} \Upsilon_{1}^{\beta, 1-\nu} \mathbf{G L}^{\beta}(x, t) \\
& +x I_{t}^{1-\nu}\left(\varphi_{1}^{\prime}(t)-\varphi_{0}^{\prime}(t)\right)+x \frac{\Gamma(1)}{\Gamma(2-\nu)} t^{1-\nu}\left(\varphi_{0}^{\prime}(0)-\varphi_{1}^{\prime}(0)\right)-x t^{2-\nu} U^{T} P_{0} \\
& \quad \times \Lambda \Theta_{0}^{\beta, 1} \Theta_{1}^{\beta, 1-\nu} L^{\beta}(t)+I_{t}^{1-\nu}\left(\varphi_{0}^{\prime}(t)\right)+\frac{\Gamma(1)}{\Gamma(2-\nu)} t^{1-\nu}\left(\omega_{1}(x)-\varphi_{0}^{\prime}(0)\right)
\end{aligned}
$$

For $1<\nu \leq 2$, we get

$$
\begin{aligned}
& D_{t}^{\nu} u(x, t)=I_{t}^{2-\nu}\left(\frac{\partial^{2} u(x, t)}{\partial t^{2}}\right) \simeq x^{2} t^{2-\nu} U^{T} P_{0} P_{1} \Upsilon_{0}^{\beta, 2-\nu} \mathbf{G L}^{\beta}(x, t) \\
& \quad+x I_{t}^{2-\nu}\left(\varphi_{1}^{\prime \prime}(t)-\varphi_{0}^{\prime \prime}(t)\right)-x t^{2-\nu} U^{T} P_{0} \Lambda \Theta_{0}^{\beta, 2-\nu} L^{\beta}(t)+I_{t}^{2-\nu}\left(\varphi_{0}^{\prime \prime}(t)\right) .
\end{aligned}
$$

Also, to approximate the fractional differential part of subdiffusion equation, we use Equation (4.5) as follows:

$$
D_{t}^{1-\gamma}\left(\frac{\partial^{2} u(x, t)}{\partial x^{2}}\right) \simeq t^{1+\gamma} U^{T} \Upsilon_{0}^{\beta, 1} \Upsilon_{1}^{\beta, 1} \Phi_{s}^{\beta, 1-\gamma} \mathbf{G L}^{\beta}(x, t)+\frac{t^{\gamma}}{\Gamma(1+\gamma)} \omega_{1}^{\prime \prime}(x)
$$

Next, we substitute the approximations results in Equations (4.1) and (4.2) to get an algebraic equation. Then, we utilize $M$ nodal points of Newton-Cotes [8] for variable $x$ and $N+1$ zeros of LPs for variable $t$, to collocate the proposed equations. Consequently, we apply Newtons method or Broydens method or any other iterative solver to get the solution of the nonlinear system.

\section{Error bound}

This section investigates the error bound of the integral POM and the error of partial derivative of the approximate solution. To compute the upper bound of 
error for POM of integration with respect to variable $x$, we use Equation (3.1), Lemmas 1 and 2 in [12], and the following relation:

$$
\begin{aligned}
& \mathbf{E}_{1, x}^{r}=\int_{0}^{x} \xi^{r} \mathbf{G L}^{\beta}(\xi, t) d \xi-x^{r+1} P_{r} \mathbf{G L}^{\beta}(x, t), \quad r=0,1,2, \ldots, \\
& \mathbf{E}_{1, x}^{r}=\left[e_{j 1}^{1, x}\right], \quad j=1,2, \ldots, M(N+1) .
\end{aligned}
$$

On the other hand, according to the above relation, we obtain

$$
\begin{aligned}
\mathbf{E}_{1, x}^{r} & =\int_{0}^{x} \xi^{r} \Psi(\xi) L^{\beta}(t) d \xi-x^{r+1} P_{r} \Psi(x) L^{\beta}(t) \\
& =\left[\int_{0}^{x} \xi^{r} \Psi(\xi) d \xi-x^{r+1} P_{r} \Psi(x)\right] L^{\beta}(t)=\mathbf{R}_{1, x}^{r} L^{\beta}(t) .
\end{aligned}
$$

Then, we get

$$
\left\|\mathbf{E}_{1, x}^{r}\right\|_{L_{w_{\beta}(x, t)}^{2}(\Omega)} \leq\left\|\mathbf{R}_{1, x}^{r}\right\|_{L^{2}[0,1]}\left\|L^{\beta}(t)\right\|_{L_{w_{\beta}(t)}^{2}[0, \infty)}
$$

where

$$
\mathbf{R}_{1, x}^{r}=\operatorname{diag}\left[\overline{\mathbf{R}}_{1, x}^{r}, \overline{\mathbf{R}}_{1, x}^{r}, \ldots, \overline{\mathbf{R}}_{1, x}^{r}\right]_{M(N+1) \times(N+1)} .
$$

Each component of $\overline{\mathbf{R}}_{1, x}^{r}$ is given by

$$
\overline{\mathbf{R}}_{1, x}^{r}=\int_{0}^{x} \xi^{r} G(\xi) d \xi-x^{r+1} \bar{P}_{r} G(x), \quad \overline{\mathbf{R}}_{1, x}^{r}=\left[\mathbf{r}_{m}^{1, x}\right], \quad m=1,2, \ldots, M .
$$

Therefore, due to the orthogonal property of fractional-order Laguerre functions in Equation (2.1), we have

$$
\left\|e_{j 1}^{1, x}\right\|_{L_{w_{\beta}(x, t)}^{2}(\Omega)} \leq \frac{1}{\sqrt{\beta}}\left\|r_{m}^{1, x}\right\|_{L^{2}[0,1]} .
$$

Moreover, from Equation (3.1) the following result is achieved:

$$
\begin{aligned}
\left\|\mathbf{r}_{m}^{1, x}\right\|_{L^{2}[0,1]} & =\left\|\int_{0}^{x} \xi^{r} G_{m}(\xi) d \xi-x^{r+1} \sum_{k=0}^{m}\left(\begin{array}{c}
m \\
k
\end{array}\right) \frac{g_{m-k}}{k+r+1}\left[\sum_{i=1}^{M} b_{k i} G_{i}(x)\right]\right\|_{L^{2}[0,1]} \\
& \leq\left|\sum_{k=0}^{m}\left(\begin{array}{c}
m \\
k
\end{array}\right) \frac{g_{m-k}}{k+r+1}\right|\left\|x^{k}-\sum_{i=1}^{M} b_{k i} G_{i}(x)\right\|_{L^{2}[0,1]} \\
& =\left|\sum_{k=0}^{m}\left(\begin{array}{c}
m \\
k
\end{array}\right) \frac{g_{m-k}}{k+r+1}\right|\left(\frac{\operatorname{Gram}\left(x^{k}, G_{1}(x), \ldots, G_{M}(x)\right)}{\operatorname{Gram}\left(G_{1}(x), \ldots, G_{M}(x)\right)}\right)^{\frac{1}{2}} .
\end{aligned}
$$

The above discussion indicates that with increasing the number of G-FLFs bases, each element of $\overline{\mathbf{R}}_{1, x}^{r}$ tends to zero quickly.

Theorem 1. Suppose that $u_{M N}(x, t)$ is the approximate of the exact solution $u(x, t)$ obtained by the proposed method over the region $\Omega$, then the upper bound 
of error is given by

$$
\begin{aligned}
\left\|\frac{\partial^{2} u(x, t)}{\partial t^{2}}-\frac{\partial^{2} u_{M N}(x, t)}{\partial t^{2}}\right\|_{L_{w_{\beta}(x, t)}^{2}(\Omega)} \leq & \left\|U^{T}\right\|_{2}\left\|\boldsymbol{E}_{2, x}^{r}\right\|_{L_{w_{\beta}(x, t)}^{2}(\Omega)} \\
& +\left\|U^{T}\right\|_{2}\left\|\boldsymbol{E}_{1, x}^{r}\right\|_{L_{w_{\beta}(x, t)}^{2}(\Omega)},
\end{aligned}
$$

where $\boldsymbol{E}_{2, x}^{r}$ is calculated as $\boldsymbol{E}_{1, x}^{r}$.

Proof. From the algorithm of the method mentioned in Section 4, the second derivative of $u(x, t)$ with respect to $t$ is expressed by

$$
\begin{aligned}
\frac{\partial^{2} u(x, t)}{\partial t^{2}}= & U^{T} \int_{0}^{x} \int_{0}^{x} \mathbf{G L}^{\beta}(\xi, t) d \xi d \xi \\
& +x\left(\varphi_{1}^{\prime \prime}(t)-\varphi_{0}^{\prime \prime}(t)-U^{T}\left(\int_{0}^{x} \mathbf{G} \mathbf{L}^{\beta}(\xi, t) d \xi\right) \Lambda L^{\beta}(t)\right)+\varphi_{0}^{\prime \prime}(t) .
\end{aligned}
$$

Considering Equation (4.9), the following expression is established:

$$
\begin{aligned}
& \left\|\frac{\partial^{2} u(x, t)}{\partial t^{2}}-\frac{\partial^{2} u_{M N}(x, t)}{\partial t^{2}}\right\|_{L_{w_{\beta}(x, t)}^{2}(\Omega)} \\
& \leq\left\|U^{T}\left[\int_{0}^{x} \int_{0}^{x} \mathbf{G L}^{\beta}(\xi, t) d \xi d \xi\right]-x^{2} U^{T} P_{0} P_{1} \mathbf{G L}^{\beta}(x, t)\right\|_{L_{w_{\beta}(x, t)}^{2}}(\Omega) \\
& \quad+\left\|x U^{T}\left[\int_{0}^{1} \int_{0}^{x} \mathbf{G L}^{\beta}(\xi, t) d \xi d x\right]-x U^{T}\left[\int_{0}^{1} x P_{0} \mathbf{G L}^{\beta}(\xi, t) d x\right]\right\|_{L_{w_{\beta}(x, t)}^{2}(\Omega)} \\
& \leq\left\|U^{T}\right\|_{2}\left\|\int_{0}^{x} \int_{0}^{x} \mathbf{G L}^{\beta}(\xi, t) d \xi d \xi-x^{2} P_{0} P_{1} \mathbf{G L}^{\beta}(x, t)\right\|_{L_{w_{\beta}(x, t)}^{2}(\Omega)} \\
& \quad+\|x\|_{L_{w_{\beta}(x, t)}^{2}(\Omega)}\left\|U^{T}\right\|_{2}\left\|\int_{0}^{1}\left[\int_{0}^{x} \mathbf{G L}^{\beta}(\xi, t) d \xi-x P_{0} \mathbf{G L}^{\beta}(x, t)\right] d x\right\|_{L_{w_{\beta}(x, t)}^{2}(\Omega)} \\
& =\left\|U^{T}\right\|_{2}\left\|\mathbf{E}_{2, x}^{r}\right\|_{L_{w_{\beta}(x, t)}^{2}(\Omega)}+\left\|U^{T}\right\|_{2}\left\|\mathbf{E}_{1, x}^{r}\right\|_{L_{w_{\beta}(x, t)}^{2}}(\Omega)
\end{aligned}
$$

From the above theorem, it can be inferred that for large values of $M$ and $N$ the upper bound of error tends to zero. In a similar manner, it can be deduced that

$$
M, N \rightarrow \infty \quad \Rightarrow \quad\left\|\frac{\partial^{3} u(x, t)}{\partial x \partial t^{2}}-\frac{\partial^{3} u_{M N}(x, t)}{\partial x \partial t^{2}}\right\|_{L_{w_{\beta}(x, t)}^{2}(\Omega)} \rightarrow 0
$$

\section{$6 \quad$ Numerical experiments}

In this section to illustrate the applicability and validity of the numerical scheme performed in the previous sections, we give seven examples. The calculations were performed on a personal computer and the codes were written in MATLAB 2016. 
Example 1. First, we consider the one-dimensional linear inhomogeneous time fractional Burgers equation [31,42]

$$
D_{t}^{\nu} u(x, t)=\frac{\partial^{2} u}{\partial x^{2}}(x, t)-\frac{\partial u}{\partial x}(x, t)+\frac{2 t^{2-\nu}}{\Gamma(3-\nu)}+2 x-2, \quad 0<\nu \leq 1,
$$

subject to the initial condition $u(x, 0)=x^{2}, x \in[0,1]$ and the boundary condition $u(0, t)=t^{2}, u(1, t)=1+t^{2}, t>0$. The exact solution is $u(x, t)=$ $x^{2}+t^{2}$. From the algorithm of the proposed approach, we obtain

$$
\begin{aligned}
\frac{\partial^{2} u(x, t)}{\partial x^{2}} & \simeq t U^{T} \Upsilon_{0}^{\beta, 1} \mathbf{G L}^{\beta}(x, t)+2, \\
\frac{\partial u(x, t)}{\partial x} & \simeq x t U^{T} \Upsilon_{0}^{\beta, 1} P_{0} \mathbf{G} \mathbf{L}^{\beta}(x, t)+2 x+1-t U^{T} \Upsilon_{0}^{\beta, 1} P_{0} \Lambda L^{\beta}(t), \\
u(x, t) & \simeq x^{2} t U^{T} \Upsilon_{0}^{\beta, 1} P_{0} P_{1} \mathbf{G L}^{\beta}(x, t)+x^{2}+x\left(1-t U^{T} \Upsilon_{0}^{\beta, 1} P_{0} \Lambda L^{\beta}(t)\right)+t^{2}, \\
D_{t}^{\nu} u(x, t) & \simeq x^{2} t^{1-\nu} U^{T} P_{0} P_{1} \Upsilon_{0}^{\beta, 1-\nu} \mathbf{G L}^{\beta}(x, t)-x t^{1-\nu} U^{T} P_{0} \Lambda \Theta_{0}^{\beta, 1-\nu} L^{\beta}(t) \\
& +\frac{2 \Gamma(2)}{\Gamma(3-\nu)} t^{2-\nu} .
\end{aligned}
$$

Then, by replacing the obtained results in Equation (6.1) and considering $M=N=2$ for various values of $\beta, \nu$, we get $u(x, t)=x^{2}+t^{2}$, which is the exact solution of Equation (6.1). The maximum absolute error on the interval $[0,1] \times[0,2]$ for $N=51$ by meshless method [31] is $5.4666 \times 10^{-5}$ and by Rbfs approximation method [31] is $6.086 \times 10^{-2}$. From the obtained results, it can be concluded that the proposed method is more accurate than the meshless method [42] and Rbfs approximation method [31].

Example 2. We consider the following fractional Klein-Gordon equation [19]

$$
D_{t}^{\nu} u(x, t)-\frac{\partial^{2} u}{\partial x^{2}}(x, t)=u(x, t), \quad 1<\nu \leq 2,
$$

subject to the initial conditions $u(x, 0)=1+\sin (x), u_{t}(x, 0)=0, x \in[0,1]$, and the boundary conditions $u(0, t)=\cosh (t), u(1, t)=\sin (1)+\cosh (t), t>0$. The exact solution of this problem is $u(x, t)=\sin (x)+\cosh (t)$ for special value of $\nu=2$. According to the mentioned approach, we obtain

$$
\begin{aligned}
\frac{\partial^{2} u(x, t)}{\partial x^{2}} \simeq & t^{2} U^{T} \Upsilon_{0}^{\beta, 1} \Upsilon_{1}^{\beta, 1} \mathbf{G L}^{\beta}(x, t)-\sin (x), \\
u(x, t) \simeq & x^{2} t^{2} U^{T} \Upsilon_{0}^{\beta, 1} \Upsilon_{1}^{\beta, 1} P_{0} P_{1} \mathbf{G} \mathbf{L}^{\beta}(x, t)-x t^{2} U^{T} \Upsilon_{0}^{\beta, 1} \Upsilon_{1}^{\beta, 1} P_{0} \Lambda L^{\beta}(t) \\
& +\sin (x)+\cosh (t), \\
D_{t}^{\nu} u(x, t) \simeq & x^{2} t^{2-\nu} U^{T} P_{0} P_{1} \Upsilon_{0}^{\beta, 2-\nu} \mathbf{G L}^{\beta}(x, t) \\
& -x t^{2-\nu} U^{T} P_{0} \Lambda \Theta_{0}^{\beta, 2-\nu} L^{\beta}(t)+\cosh (t)
\end{aligned}
$$

By taking $\nu=2, M=N=2$ and replacing the aforesaid relation in Equation (6.2), we get $u(x, t)=\sin (x)+\cosh (t)$, which is the exact solution of the 
Table 1. Absolute error for different values of $\nu$ with $\beta=1$ and $M=N=2$ on the interval $[0,1] \times[0,5]$ for Example 2 .

\begin{tabular}{ccccc}
\hline$(x, t)$ & $\nu=1.8$ & $\nu=1.85$ & $\nu=1.9$ & $\nu=1.95$ \\
\hline$(0,0)$ & 0 & 0 & 0 & 0 \\
$(0.2,1)$ & $1.05 \times 10^{-2}$ & $7.30 \times 10^{-3}$ & $4.45 \times 10^{-3}$ & $2.00 \times 10^{-3}$ \\
$(0.4,2)$ & $3.79 \times 10^{-2}$ & $2.63 \times 10^{-2}$ & $1.60 \times 10^{-2}$ & $7.19 \times 10^{-3}$ \\
$(0.6,3)$ & $2.86 \times 10^{-2}$ & $1.79 \times 10^{-2}$ & $1.19 \times 10^{-2}$ & $5.31 \times 10^{-3}$ \\
$(0.8,4)$ & $3.33 \times 10^{-2}$ & $2.33 \times 10^{-2}$ & $1.44 \times 10^{-2}$ & $6.57 \times 10^{-3}$ \\
$(1,5)$ & 0 & 0 & 0 & 0 \\
\hline \multicolumn{7}{r}{} & & &
\end{tabular}
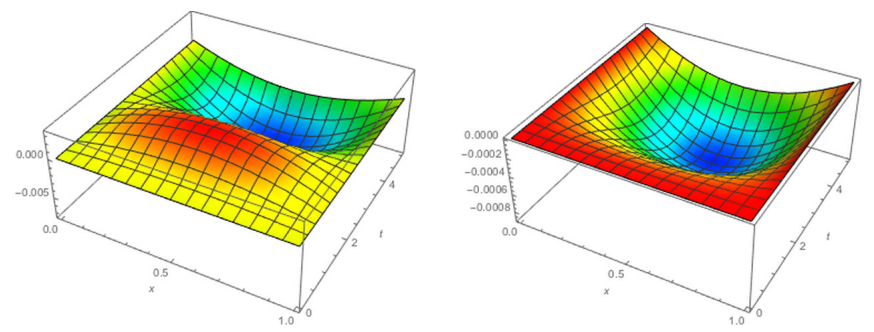

Figure 1. The absolute errors of $\nu=1.75$ (left) and $\nu=1.95$ (right) with $\beta=0.5$ and $M=4, N=4$ on the interval $[0,1] \times[0,5]$ of Example 2.

present example. From Table 1, it is clear that with approaching the values of $\nu$ to 2 the approximate solution tends to the exact solution.

Also, the absolute errors for the different choice of $\nu$ with $\beta=0.5$ and $M=N=4$ on the interval $[0,1] \times[0,5]$ are demonstrated in Figure 1.

Example 3. Let us consider the following two-term wave-diffusion equation with damping $[5,28,38]$ :

$$
D_{t}^{\nu} u(x, t)+\frac{\partial u}{\partial t}(x, t)=\frac{\partial^{2} u}{\partial x^{2}}(x, t)+\left(\frac{6 t^{3-\nu}}{\Gamma(4-\nu)}+3 t^{2}-t^{3}\right) e^{x}, \quad 1<\nu \leq 2
$$

subject to the initial conditions $u(x, 0)=0, u_{t}(x, 0)=0, x \in[0,1]$ and the boundary conditions $u(0, t)=t^{3}, u(1, t)=e t^{3}, t>0$. The exact solution is $u(x, t)=t^{3} e^{x}$. Table 2 represents the absolute errors by the present technique and methods in $[5,28,38]$. From Table 2, we conclude that the results produced by our technique with a few terms of G-FLFs are more accurate than methods in $[5,28,38]$. Also, the absolute errors for $\nu=1.5$ and $\beta=1$ on interval $[0,1] \times[0,50]$ are plotted in Figure 2. It illustrates that the proposed method has high precision in the large interval.

Example 4. We consider the time-fractional sine-Gordon equation with the following form $[8,14,16]$ :

$$
D_{t}^{\nu} u(x, t)=\frac{\partial^{2} u}{\partial x^{2}}(x, t)-\sin (u(x, t))+f(x, t), \quad 1<\nu \leq 2,
$$


Table 2. Comparison of the maximum absolute errors for different values of $M$ with methods in $[5,28,38]$ with $\nu=1.85$ and $\beta=1$ on the interval $[0,1] \times[0,1]$ for Example 3 .

\begin{tabular}{ccccc}
\hline Method & $M$ & $N$ & $L_{\infty}-$ error & $C P U$ \\
\hline Present method & 2 & 2 & $4.1933 \times 10^{-4}$ & 0.1762 \\
& 4 & 2 & $7.4878 \times 10^{-7}$ & 0.2152 \\
& 8 & 2 & $8.4505 \times 10^{-13}$ & 0.3147 \\
\hline Semi-analytical collocation method [38] & 4 & 4 & $3.38 \times 10^{-3}$ & - \\
& 8 & 8 & $9.69 \times 10^{-4}$ & - \\
& 16 & 16 & $2.53 \times 10^{-4}$ & - \\
\hline Jacobi tau spectral method [5] & 4 & 4 & $5.46 \times 10^{-5}$ & - \\
& 8 & 8 & $5.50 \times 10^{-6}$ & - \\
& 16 & 16 & $2.42 \times 10^{-7}$ & - \\
\hline Predictor-corrector method [28] & 4 & 4 & $1.09 \times 10^{-1}$ & - \\
& 8 & 8 & $2.76 \times 10^{-2}$ & - \\
& 16 & 16 & $6.72 \times 10^{-3}$ & - \\
\hline
\end{tabular}
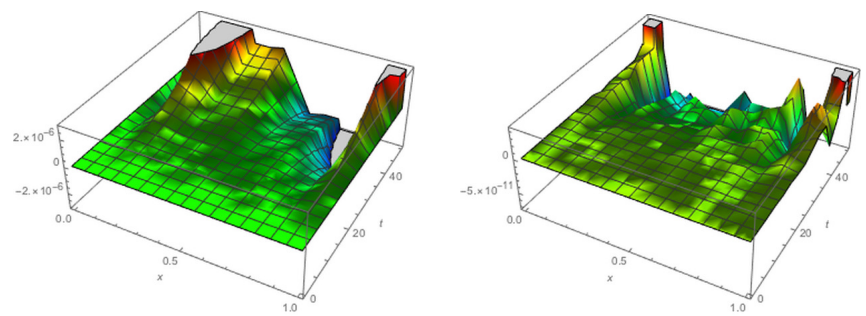

Figure 2. The absolute errors for $\nu=1.5$ and $\beta=1$ with $M=7, N=2$ (left) and $M=10, N=2$ (right) on the interval $[0,1] \times[0,50]$ of Example 3.

subject to the initial conditions $u(x, 0)=0, u_{t}(x, 0)=0, x \in[0,1]$ and the boundary conditions $u(0, t)=0, u(1, t)=t^{2} \sin (1), t>0$, where $f(x, t)=$ $\left(\frac{2 t^{2-\nu}}{\Gamma(3-\nu)}+t^{2}\right) \sin (x)+\sin \left(t^{2} \sin (x)\right)$. The exact solutions is $u(x, t)=t^{2} \sin (x)$.

Table 3. Absolute error for different values of $M$ with $\beta=1$ and $\nu=2$ on the interval $[0,1] \times[0,10]$ for Example 4 .

\begin{tabular}{lcccc}
\hline$(x, t)$ & $N=2, M=3$ & $N=2, M=5$ & $N=2, M=7$ & $N=2, M=10$ \\
\hline$(0,0)$ & 0 & 0 & 0 & 0 \\
$(0.2,2)$ & $4.2101 \times 10^{-5}$ & $1.6382 \times 10^{-7}$ & $1.3178 \times 10^{-10}$ & $3.7485 \times 10^{-16}$ \\
$(0.4,4)$ & $3.2681 \times 10^{-4}$ & $1.0324 \times 10^{-8}$ & $1.9122 \times 10^{-10}$ & $2.1658 \times 10^{-17}$ \\
$(0.6,6)$ & $6.5825 \times 10^{-4}$ & $1.4881 \times 10^{-7}$ & $3.5734 \times 10^{-10}$ & $1.3180 \times 10^{-14}$ \\
$(0.8,8)$ & $7.2652 \times 10^{-4}$ & $2.6302 \times 10^{-6}$ & $2.2944 \times 10^{-9}$ & $3.7462 \times 10^{-14}$ \\
$(1,10)$ & $1.7768 \times 10^{-16}$ & $1.7768 \times 10^{-16}$ & $1.7768 \times 10^{-16}$ & $1.7768 \times 10^{-16}$ \\
\hline
\end{tabular}

In Table 3, we present the absolute errors for different values of $M$ with 
$N=2, \beta=1$ and $\nu=2$ on the interval $[0,1] \times[0,10]$. Table 3 indicates that with the increase in the number of G-FLFs bases the error decreases sharply.

Moreover, we observe the comparison of the absolute errors by G-FLFs for different choices of $\beta$ and methods in $[14,16]$, in Table 4.

Table 4. Comparison of the absolute errors obtained by presented approach for various values of $\beta$ and $M=8, N=2$ with method in $[14,16]$ of Example 4 .

\begin{tabular}{|c|c|c|c|c|}
\hline \multirow[t]{2}{*}{$x=t$} & \multicolumn{2}{|c|}{ Present method } & \multirow{2}{*}{$\begin{array}{c}\text { Method in [14] } \\
M=N=8\end{array}$} & \multirow{2}{*}{$\begin{array}{l}\text { Method in }[16] \\
M=N=8\end{array}$} \\
\hline & $\beta=0.5$ & $\beta=1$ & & \\
\hline$\frac{1}{10}$ & $3.3232 \times 10^{-15}$ & $3.6083 \times 10^{-15}$ & $9.83 \times 10^{-3}$ & $7.79 \times 10^{-14}$ \\
\hline$\frac{1}{20}$ & $1.8092 \times 10^{-15}$ & $1.7705 \times 10^{-15}$ & $4.59 \times 10^{-3}$ & $2.17 \times 10^{-14}$ \\
\hline$\frac{20}{40}$ & $6.3314 \times 10^{-16}$ & $6.2835 \times 10^{-16}$ & $2.24 \times 10^{-3}$ & $9.43 \times 10^{-16}$ \\
\hline$\frac{1}{80}$ & $1.1921 \times 10^{-16}$ & $1.1863 \times 10^{-16}$ & $1.11 \times 10^{-3}$ & $6.57 \times 10^{-16}$ \\
\hline$\frac{1}{160}$ & $1.8000 \times 10^{-17}$ & $1.7928 \times 10^{-17}$ & $5.52 \times 10^{-4}$ & $1.21 \times 10^{-16}$ \\
\hline$C P U$ & 0.3451 & 0.3128 & - & - \\
\hline
\end{tabular}
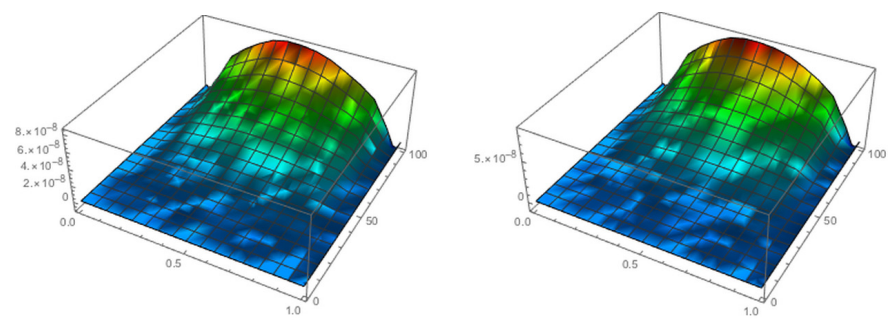

Figure 3. The absolute error for $\nu=1.75$ (left) and $\nu=1.95$ (right) with $M=8, N=2$ and $\beta=1$ on the interval $[0,1] \times[0,100]$ of Example 4 .

In Figure 3, the absolute errors are illustrated by considering the points in the interval $[0,1] \times[0,100]$ for different values of $\nu=1.75,1.95$ and $\beta=1, M=$ $2, N=8$. Due to the obtained results and results of the method in [8], it can be concluded that the present method is more accurate than the mentioned method.

Example 5. Consider the following subdiffusion equation $[45,47]$ :

$$
\frac{\partial u}{\partial t}(x, t)=D_{t}^{1-\gamma}\left(\frac{\partial^{2} u}{\partial x^{2}}(x, t)\right)+f(x, t), \quad 0<\gamma<1
$$

subject to the initial conditions $u(x, 0)=\exp (x), x \in[0,1]$ and the boundary conditions $u(0, t)=t^{2}+t+1, u(1, t)=\exp (1)\left(t^{2}+t+1\right), t>0$, where $f(x, t)$ is defined such that the exact solution of this example is $u(x, t)=\exp (x)\left(t^{2}+t+1\right)$.

The absolute error for various values of $M, N, \beta, \gamma$ on the interval $[0,1] \times$ $[0,100]$ are presented in Table 5 . 
Table 5. Absolute errors for different values of $M, N, \beta, \gamma$ on the interval $[0,1] \times[0,100]$ for Example 5.

\begin{tabular}{ccccc}
\hline$(x, t)$ & \multicolumn{2}{c}{$M=5, N=3, \beta=1$} & \multicolumn{2}{c}{$M=5, N=5, \beta=0.5$} \\
& $\gamma=0.25$ & $\gamma=0.5$ & $\gamma=0.25$ & $\gamma=0.5$ \\
\hline$(0,0)$ & 0 & 0 & 0 & 0 \\
$(0.1,10)$ & $7.3360 \times 10^{-7}$ & $7.2895 \times 10^{-7}$ & $7.3393 \times 10^{-10}$ & $7.2881 \times 10^{-7}$ \\
$(0.2,20)$ & $3.0724 \times 10^{-5}$ & $3.0772 \times 10^{-5}$ & $3.0704 \times 10^{-5}$ & $3.0756 \times 10^{-5}$ \\
$(0.3,30)$ & $3.3782 \times 10^{-5}$ & $3.3883 \times 10^{-5}$ & $3.3674 \times 10^{-5}$ & $3.3794 \times 10^{-5}$ \\
$(0.4,40)$ & $1.3335 \times 10^{-5}$ & $1.3289 \times 10^{-5}$ & $1.3474 \times 10^{-5}$ & $1.3392 \times 10^{-5}$ \\
$(0.5,50)$ & $6.2643 \times 10^{-6}$ & $6.4562 \times 10^{-6}$ & $5.9209 \times 10^{-6}$ & $6.0693 \times 10^{-6}$ \\
$(0.6,60)$ & $1.0418 \times 10^{-5}$ & $9.8118 \times 10^{-6}$ & $1.2239 \times 10^{-5}$ & $1.1795 \times 10^{-5}$ \\
$(0.7,70)$ & $2.1813 \times 10^{-4}$ & $2.1890 \times 10^{-4}$ & $2.1412 \times 10^{-4}$ & $2.1469 \times 10^{-4}$ \\
$(0.8,80)$ & $5.2874 \times 10^{-4}$ & $5.2947 \times 10^{-4}$ & $5.2355 \times 10^{-4}$ & $5.2395 \times 10^{-4}$ \\
$(0.9,90)$ & $8.5065 \times 10^{-5}$ & $8.4643 \times 10^{-5}$ & $8.8203 \times 10^{-5}$ & $8.8102 \times 10^{-5}$ \\
$(1,100)$ & $3.0251 \times 10^{-12}$ & $3.0251 \times 10^{-12}$ & $3.0251 \times 10^{-12}$ & $3.0251 \times 10^{-12}$ \\
\hline
\end{tabular}

Table 6. Comparison of the $L_{2}$ and $L_{\infty}$ errors for different values of $M, \gamma$ and $\beta=1$ with methods in [45] on the interval $[0,1] \times[0,1]$ for Example 5 .

\begin{tabular}{cccccc}
\hline Method & $\gamma$ & $M$ & $N$ & $L_{2}$-error & $L_{\infty}$-error \\
\hline Present method & 0.25 & 3 & 3 & $8.9419 \times 10^{-5}$ & $3.9728 \times 10^{-5}$ \\
& & 5 & 3 & $1.2958 \times 10^{-7}$ & $1.1557 \times 10^{-7}$ \\
& & 7 & 3 & $1.0903 \times 10^{-10}$ & $9.3169 \times 10^{-11}$ \\
\cline { 2 - 6 } & 0.75 & 3 & 3 & $8.9272 \times 10^{-5}$ & $5.8494 \times 10^{-5}$ \\
& & 5 & 3 & $1.2904 \times 10^{-7}$ & $1.1517 \times 10^{-7}$ \\
Crank-Nicolson finite element & 0.25 & - & 16 & $1.3462 \times 10^{-4}$ & $1.8404 \times 10^{-4}$ \\
method [45] & & - & 32 & $3.4450 \times 10^{-5}$ & $4.7114 \times 10^{-5}$ \\
& & - & 64 & $9.4175 \times 10^{-6}$ & $1.2889 \times 10^{-5}$ \\
\cline { 2 - 6 } & 0.75 & - & 16 & $1.3615 \times 10^{-4}$ & $1.8612 \times 10^{-4}$ \\
& & - & 32 & $3.4028 \times 10^{-5}$ & $4.6532 \times 10^{-5}$ \\
& & - & 64 & $8.5093 \times 10^{-6}$ & $1.1645 \times 10^{-5}$ \\
\hline
\end{tabular}

The root-mean-square error $L_{2}$ and maximum absolute error $L_{\infty}$ in some nodes on interval $[0,1] \times[0,1]$ for different choices of $\gamma, M$ with $N=3, \beta=1$ are listed in Table 6.

Also, Table 6 observed that the proposed method is more accurate than the Crank-Nicolson finite element method [45]. Figure 4 shows the behavior of the absolute error on the interval $[0,1] \times[0,200]$ for $\gamma=0.25$ (left) and $\gamma=0.5$ (right) with $M=10, N=3$ and $\beta=1$.

Example 6. Consider the following subdiffusion equation $[7,17,20]$ :

$$
\frac{\partial u}{\partial t}(x, t)=D_{t}^{1-\gamma}\left(\frac{\partial^{2} u}{\partial x^{2}}(x, t)\right)+\exp (x)\left((1+\gamma) t^{\gamma}-\frac{\Gamma(2+\gamma)}{\Gamma(1+2 \gamma)} t^{2 \gamma}\right), 0<\gamma<1
$$

subject to the initial conditions $u(x, 0)=0, x \in[0,1]$ and the boundary conditions $u(0, t)=t^{1+\gamma}, u(1, t)=\exp (1) t^{1+\gamma}, t>0$, The exact solution 

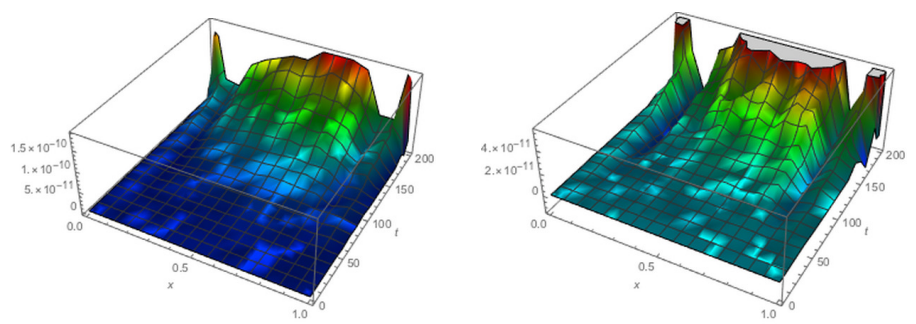

Figure 4. The absolute errors for $\gamma=0.25$ (left) and $\gamma=0.5$ (right) with $M=10, N=3$ and $\beta=1$ on the interval $[0,1] \times[0,200]$ of Example 5 .

of this example is $u(x, t)=\exp (x) t^{1+\gamma}$. We implement the suggested method for different values of $M, N, \beta, \gamma$ on the interval $[0,1] \times[0,1]$ in Table 7 .

Table 7. Comparison of the absolute errors for different values of $M, N, \beta, \gamma$ with method in $[20]$ on the interval $[0,1] \times[0,1]$ for Example 6 .

\begin{tabular}{|c|c|c|c|c|}
\hline \multirow[b]{3}{*}{$x=t$} & \multicolumn{4}{|c|}{ Present method } \\
\hline & \multicolumn{2}{|c|}{$\beta=\gamma=0.25$} & \multicolumn{2}{|c|}{$\beta=\gamma=0.5$} \\
\hline & $M=2, N=6$ & $M=5, N=6$ & $M=2, N=4$ & $M=4, N=4$ \\
\hline 0.1 & $1.09 \times 10^{-5}$ & $1.17 \times 10^{-8}$ & $8.38 \times 10^{-6}$ & $1.06 \times 10^{-8}$ \\
\hline 0.3 & $2.55 \times 10^{-4}$ & $7.94 \times 10^{-7}$ & $1.78 \times 10^{-4}$ & $4.16 \times 10^{-7}$ \\
\hline 0.5 & $8.51 \times 10^{-4}$ & $4.13 \times 10^{-7}$ & $6.99 \times 10^{-4}$ & $3.08 \times 10^{-7}$ \\
\hline 0.7 & $6.91 \times 10^{-4}$ & $1.84 \times 10^{-6}$ & $6.17 \times 10^{-4}$ & $1.65 \times 10^{-6}$ \\
\hline 0.9 & $3.22 \times 10^{-4}$ & $3.29 \times 10^{-7}$ & $3.20 \times 10^{-4}$ & $3.33 \times 10^{-7}$ \\
\hline $\mathrm{CPU}$ & 0.1563 & 0.2061 & 0.1917 & 0.3035 \\
\hline & Wavelets Gale & in method [20] & & \\
\hline & $\gamma=0.25$ & $\gamma=0.5$ & & \\
\hline$x=t$ & $k=2, M=12$ & $k=2, M=12$ & & \\
\hline 0.1 & $4.4010 \times 10^{-6}$ & $5.8425 \times 10^{-6}$ & & \\
\hline 0.3 & $3.0210 \times 10^{-6}$ & $7.4888 \times 10^{-6}$ & & \\
\hline 0.5 & $7.0767 \times 10^{-7}$ & $5.7963 \times 10^{-6}$ & & \\
\hline 0.7 & $1.4172 \times 10^{-6}$ & $2.7013 \times 10^{-6}$ & & \\
\hline 0.9 & $1.6218 \times 10^{-6}$ & $5.9588 \times 10^{-8}$ & & \\
\hline
\end{tabular}

The results of the present approach with wavelet Galerkin method [20] are compared in Table 7. It is clear that our method with a few numbers of G-FLFs is more accurate than wavelets Galerkin method. The author in [7] presented the maximum absolute errors for different cases $\gamma$ by high-order compact finite difference method. Therefore, $\gamma=0.25$ and $\gamma=0.75$ are obtained $1.8928 \times 10^{-5}$ and $5.6363 \times 10^{-5}$, respectively. Also, Gao and Sun [17] presented the maximum absolute errors by compact finite difference scheme for $\gamma=0.25$ and $\gamma=0.75$ are $1.4338 \times 10^{-8}$ and $7.1085 \times 10^{-6}$, respectively. In addition, the graph of absolute error for different values of $M$ and $N$ with $\beta=0.5, \gamma=0.25$ are plotted in Figure 5. From the obtained results, we can conclude that the mentioned 
approach is in excellent agreement with the exact solution.
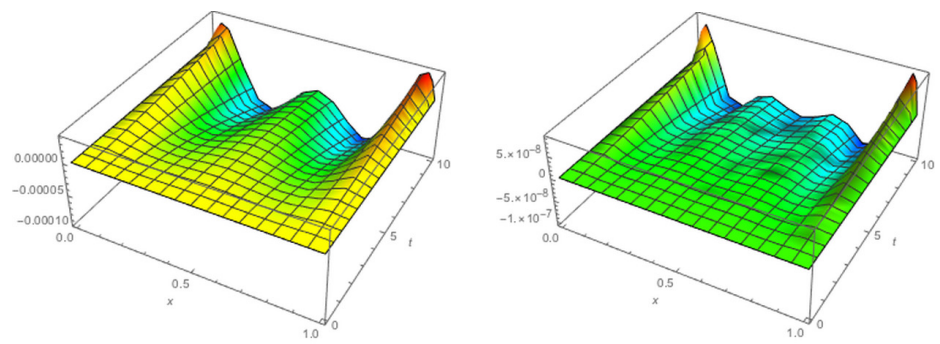

Figure 5. The absolute errors for $\beta=0.5$ and $\gamma=0.25$ with $M=4, N=6$ (left) and $M=6, N=6$ (right) on the interval $[0,1] \times[0,10]$ of Example 6 .

Example 7. We consider the following nonlinear generalized Benjamin-BonaMahony-Burgers equation:

$\frac{\partial u}{\partial t}(x, t)-D_{t}^{1-\gamma}\left(\frac{\partial^{2} u}{\partial x^{2}}(x, t)\right)-\frac{\partial^{2} u}{\partial x^{2}}(x, t)+\frac{\partial u}{\partial x}(x, t)=u(x, t) \frac{\partial u}{\partial x}(x, t)+f(x, t)$

$0<\gamma \leq 1$, subject to the initial condition $u(x, 0)=\sec h(x), x \in[0,1]$ and the boundary conditions $u(0, t)=\sec h(-t), u(1, t)=\sec h(1-t), t>0$, where $f(x, t)$ is defined such that the exact solution of this example is $u(x, t)=$ $\sec h(x-t)$. The behavior of the absolute error of this problem for different values of $\gamma$ with $M=N=5$ are expressed in Table 8 .

Table 8. Absolute errors for various values of $\gamma$ with $\beta=1$ and $M=N=5$ on the interval $[0,1] \times[0,1]$ for Example 7 .

\begin{tabular}{cccccc}
\hline$x=t$ & $\gamma=0.8$ & $\gamma=0.85$ & $\gamma=0.9$ & $\gamma=0.95$ & $\gamma=1$ \\
\hline 0 & 0 & 0 & 0 & 0 & 0 \\
0.1 & $2.7218 \times 10^{-6}$ & $2.2850 \times 10^{-6}$ & $1.8066 \times 10^{-6}$ & $1.2749 \times 10^{-6}$ & $6.7496 \times 10^{-7}$ \\
0.3 & $1.6089 \times 10^{-5}$ & $1.3164 \times 10^{-5}$ & $9.9425 \times 10^{-6}$ & $6.3449 \times 10^{-6}$ & $2.2667 \times 10^{-6}$ \\
0.5 & $3.0084 \times 10^{-5}$ & $2.4436 \times 10^{-5}$ & $1.8196 \times 10^{-5}$ & $1.1211 \times 10^{-5}$ & $3.2766 \times 10^{-6}$ \\
0.7 & $3.7621 \times 10^{-5}$ & $3.1051 \times 10^{-5}$ & $2.3785 \times 10^{-5}$ & $1.5645 \times 10^{-5}$ & $6.3977 \times 10^{-6}$ \\
0.9 & $2.4285 \times 10^{-5}$ & $2.0655 \times 10^{-5}$ & $1.6640 \times 10^{-5}$ & $1.2144 \times 10^{-5}$ & $7.0420 \times 10^{-6}$ \\
1 & 0 & 0 & 0 & 0 & 0
\end{tabular}

The given results illustrate that proposed approximate solution converges to the exact solution when $\gamma$ approaches 1 . The root-mean-square error $L_{2}$ and maximum absolute error $L_{\infty}$ on interval $[0,1] \times[0,1]$ for various values of $h$ and $M=N=5$ are listed in Table 9 .

Table 10 displays the absolute error for various values of $\beta$ with $\gamma=1, M=$ $N=3$ and $h=\frac{1}{10}$ on interval $[0,1] \times[0,10]$. This table demonstrates that the presented method is efficient for different choices of $\beta$. 
Table 9. Errors for various values of $h$ with $\gamma=1$ and $M=N=5$ on the interval $[0,1] \times[0,1]$ for Example 7 .

\begin{tabular}{ccc}
\hline$h$ & $L_{2}$-error & $L_{\infty}$-error \\
\hline $1 / 10$ & $1.4149 \times 10^{-5}$ & $7.9337 \times 10^{-6}$ \\
$1 / 20$ & $2.4886 \times 10^{-5}$ & $1.1646 \times 10^{-5}$ \\
$1 / 30$ & $1.1447 \times 10^{-5}$ & $5.5130 \times 10^{-6}$ \\
$1 / 40$ & $6.5008 \times 10^{-6}$ & $3.1302 \times 10^{-6}$ \\
\hline
\end{tabular}

Table 10. Absolute errors for various values of $\beta$ with $\gamma=1, M=N=3$ and $h=\frac{1}{10}$ on the interval $[0,1] \times[0,10]$ for Example 7 .

\begin{tabular}{ccccc}
\hline$(x, t)$ & $\beta=0.25$ & $\beta=0.5$ & $\beta=0.75$ & $\beta=1$ \\
\hline$(0.1,1)$ & $9.5033 \times 10^{-5}$ & $8.7834 \times 10^{-5}$ & $8.9462 \times 10^{-5}$ & $9.4025 \times 10^{-5}$ \\
$(0.3,3)$ & $1.6723 \times 10^{-3}$ & $1.6723 \times 10^{-3}$ & $1.6717 \times 10^{-3}$ & $1.6686 \times 10^{-3}$ \\
$(0.5,5)$ & $5.4746 \times 10^{-3}$ & $5.4723 \times 10^{-3}$ & $5.4741 \times 10^{-3}$ & $5.4813 \times 10^{-3}$ \\
$(0.7,7)$ & $9.3681 \times 10^{-3}$ & $9.3866 \times 10^{-3}$ & $9.3633 \times 10^{-3}$ & $9.2682 \times 10^{-3}$ \\
$(0.9,9)$ & $7.3145 \times 10^{-3}$ & $7.3535 \times 10^{-3}$ & $7.2919 \times 10^{-3}$ & $7.0379 \times 10^{-3}$ \\
$(1,10)$ & $2.8480 \times 10^{-40}$ & $2.6884 \times 10^{-40}$ & $2.6864 \times 10^{-40}$ & $2.7345 \times 10^{-40}$ \\
\hline
\end{tabular}

\section{Conclusions}

In this work, a Genocchi-fractional Laguerre collocation method was applied successfully for the numerical solution of FDEs and FSDEs. Then, we introduced the pseudo-operational matrices in implementation of the method. The great advantages of the presented scheme are the novel G-FLFs, the pseudooperational matrices with high accuracy, and quick reduction of FDEs and FSDEs to systems of nonlinear algebraic equations. The outcomes illustrate that the computed solutions for various values of $\beta$ have good agreement with the exact solution. From the tables and graphs plotted, it can be inferred that by applying a small value of G-FLFs the satisfactory results with high accuracy is obtained.

\section{Acknowledgements}

This work is supported by Alzahra University. We express our sincere thanks to the anonymous referees for their valuable suggestions that improved the final manuscript.

\section{References}

[1] O.P. Agrawal. A general formulation and solution scheme for fractional optimal control problems. Nonlinear Dynamics, 38(1-4):323-337, 2004. https://doi.org/10.1007/s11071-004-3764-6. 
[2] A. Aminataei and S.K. Vanani. Numerical solution of fractional Fokker-Planck equation using the operational collocation method. Applied Mathematics and Computation, 12(1):33-43, 2013.

[3] I. Aziz, S. Islamb and M. Asif. Haar wavelet collocation method for three-dimensional elliptic partial differential equations. Computers \& Mathematics with Applications, $\mathbf{7 3 ( 9 ) : 2 0 2 3 - 2 0 3 4 ,} 2017$. https://doi.org/10.1016/j.camwa.2017.02.034.

[4] A. Bhardwaj and A. Kumar. A meshless method for time fractional nonlinear mixed diffusion and diffusion-wave equation. Applied Numerical Mathematics, 160:146-165, 2021. https://doi.org/10.1016/j.apnum.2020.09.019.

[5] A.H. Bhrawy, E.H. Doha, D. Baleanu and S.S. Ezz-Eldien. A spectral tau algorithm based on Jacobi operational matrix for numerical solution of time fractional diffusion-wave equations. Journal of Computational Physics, 293:142-156, 2015. https://doi.org/10.1016/j.jcp.2014.03.039.

[6] A.H. Bhrawy and M.A. Zaky. Shifted fractional-order Jacobi orthogonal functions: application to a system of fractional differential equations. Applied Mathematical Modelling, 40(2):832-845, 2016. https://doi.org/10.1016/j.apm.2015.06.012.

[7] M. Cui. Compact finite difference method for the fractional diffusion equation. Journal of Computational Physics, 228(20):7792-7804, 2009. https://doi.org/10.1016/j.jcp.2009.07.021.

[8] H. Dehestani, Y. Ordokhani and M. Razzaghi. Fractional-order LegendreLaguerre functions and their applications in fractional partial differential equations. Applied Mathematics and Computation, 336:433-453, 2018. https://doi.org/10.1016/j.amc.2018.05.017.

[9] H. Dehestani, Y. Ordokhani and M. Razzaghi. Hybrid functions for numerical solution of fractional Fredholm-Volterra functional integro-differential equations with proportional delays. International Journal of Numerical Modelling: Electronic Networks, Devices and Fields, 32(5):e2606, 2019. https://doi.org/10.1002/jnm.2606.

[10] H. Dehestani, Y. Ordokhani and M. Razzaghi. A numerical technique for solving various kinds of fractional partial differential equations via Genocchi hybrid functions. Revista de la Real Academia de Ciencias Exactas, Físicas y Naturales. Serie A. Matemáticas, 113(4):3297-3321, 2019. https://doi.org/10.1007/s13398019-00694-5.

[11] H. Dehestani, Y. Ordokhani and M. Razzaghi. A novel direct method based on the Lucas multiwavelet functions for variable-order fractional reactiondiffusion and subdiffusion equations. Numerical Linear Algebra with Applications, 28(2):e2346, 2020. https://doi.org/10.1002/nla.2346.

[12] H. Dehestani, Y. Ordokhani and M. Razzaghi. The novel operational matrices based on 2D-Genocchi polynomials: solving a general class of variable-order fractional partial integro-differential equations. Computational and Applied Mathematics, 39(4):1-32, 2020. https://doi.org/10.1007/s40314-020-01314-4.

[13] M. Dehghan, M. Abbaszadeh and A. Mohebbi. The numerical solution of nonlinear high dimensional generalized Benjamin-Bona-MahonyBurgers equation via the meshless method of radial basis functions. Computers \& Mathematics with Applications, 68(3):212-237, 2014. https://doi.org/10.1016/j.camwa.2014.05.019. 
[14] M. Dehghan, M. Abbaszadeh and A. Mohebbi. An implicit RBF meshless approach for solving the time fractional nonlinear sine-Gordon and Klein-Gordon equations. Engineering Analysis with Boundary Elements, 50:412-434, 2015. https://doi.org/10.1016/j.enganabound.2014.09.008.

[15] M. Dehghan, E.A. Hamedi and H. Khosravian-Arab. A numerical scheme for the solution of a class of fractional variational and optimal control problems using the modified Jacobi polynomials. Journal of Vibration and Control, 22(6):15471559, 2016. https://doi.org/10.1177/1077546314543727.

[16] E.H. Doha, M.A. Abdelkawy, A.Z.M. Amin and A. Lopes. A space-time spectral approximation for solving nonlinear variable-order fractional sine and Klein-Gordon differential equations. Computational and Applied Mathematics, 37(5):6212-6229, 2018. https://doi.org/10.1007/s40314-018-0695-2.

[17] G. Gao and Z. Sun. A compact finite difference scheme for the fractional subdiffusion equations. Journal of Computational Physics, 230(3):586-595, 2011. https://doi.org/10.1016/j.jcp.2010.10.007.

[18] R. Ghaffari and F. Ghoreishi. Reduced spline method based on a proper orthogonal decomposition technique for fractional subdiffusion equations. Applied Numerical Mathematics, 137:62-79, 2019. https://doi.org/10.1016/j.apnum.2018.11.014.

[19] A.K. Golmankhaneh, A.K. Golmankhaneh and D. Baleanu. On nonlinear fractional Klein-Gordon equation. Signal Processing, 91(3):446-451, 2011. https://doi.org/10.1016/j.sigpro.2010.04.016.

[20] M.H. Heydari. Wavelets Galerkin method for the fractional subdiffusion equation. Journal of Computational and Nonlinear Dynamics, 11(6), 2016. https://doi.org/10.1115/1.4034391.

[21] A. Isah and C. Phang. Operational matrix based on Genocchi polynomials for solution of delay differential equations. Ain Shams Engineering Journal, 9(4):2123-2128, 2018. https://doi.org/10.1016/j.asej.2016.09.015.

[22] A. Isah and C. Phang. New operational matrix of derivative for solving non-linear fractional differential equations via Genocchi polynomials. Journal of King Saud University-Science, 31(1):1-7, 2019. https://doi.org/10.1016/j.jksus.2017.02.001.

[23] Y. Jiang and J. Ma. High-order finite element methods for time-fractional partial differential equations. Journal of Computational and Applied Mathematics, 235(11):3285-3290, 2011. https://doi.org/10.1016/j.cam.2011.01.011.

[24] A. Kumar, A. Bhardwaj and S. Dubey. A local meshless method to approximate the time-fractional telegraph equation. Engineering with Computers, 37:34733488, 2021. https://doi.org/10.1007/s00366-020-01006-x.

[25] A. Kumar, A. Bhardwaj and B.V. Rathish Kumar. A meshless local collocation method for time fractional diffusion wave equation. Computers \& Mathematics with Applications, 78(6):1851-1861, 2019. https://doi.org/10.1016/j.camwa.2019.03.027.

[26] S. Kumar and C. Piret. Numerical solution of space-time fractional PDEs using RBF-QR and Chebyshev polynomials. Applied Numerical Mathematics, 143:300-315, 2019. https://doi.org/10.1016/j.apnum.2019.04.012.

[27] C. Li, T. Zhao, W. Deng and Y. Wu. Orthogonal spline collocation methods for the subdiffusion equation. Journal of Computational and Applied Mathematics, 255:517-528, 2014. https://doi.org/10.1016/j.cam.2013.05.022. 
[28] F. Liu, M. Meerschaert, Ri McGough, P. Zhuang and Q. Liu. Numerical methods for solving the multi-term time-fractional wave-diffusion equation. Fractional Calculus and Applied Analysis, 16(1):9-25, 2013. https://doi.org/10.2478/s13540-013-0002-2.

[29] J.R. Loh, C. Phang and A. Isah. New operational matrix via Genocchi polynomials for solving Fredholm-Volterra fractional integrodifferential equations. Advances in Mathematical Physics, 2017, 2017. https://doi.org/10.1155/2017/3821870.

[30] R.L. Magin. Fractional calculus models of complex dynamics in biological tissues. Computers \& Mathematics with Applications, 59(5):1586-1593, 2010. https://doi.org/10.1016/j.camwa.2009.08.039.

[31] A. Mardani, M.R. Hooshmandasl, M.H. Heydari and C. Cattani. A meshless method for solving the time fractional advection-diffusion equation with variable coefficients. Computers $\&$ Mathematics with Applications, 75(1):122-133, 2018. https://doi.org/10.1016/j.camwa.2017.08.038.

[32] R. Metzler and J. Klafter. The random walk's guide to anomalous diffusion: a fractional dynamics approach. Physics reports, 339(1):1-77, 2000. https://doi.org/10.1016/S0370-1573(00)00070-3.

[33] N. Mollahasani, M.M. Moghadam and K. Afrooz. A new treatment based on hybrid functions to the solution of telegraph equations of fractional order. Applied Mathematical Modelling, 40(4):2804-2814, 2016. https://doi.org/10.1016/j.apm.2015.08.020.

[34] K. Mustapha. An implicit finite-difference time-stepping method for a sub-diffusion equation, with spatial discretization by finite elements. IMA Journal of Numerical Analysis, 31(2):719-739, 2011. https://doi.org/10.1093/imanum/drp057.

[35] M.D. Ortigueira and J.A.T. Machado. Fractional signal processing and applications. Signal processing, 83(11):2285-2286, 2003. https://doi.org/10.1016/S0165-1684(03)00181-6.

[36] J. Ren, Z.Z. Sun and X. Zhao. Compact difference scheme for the fractional sub-diffusion equation with Neumann boundary conditions. Journal of Computational Physics, 232(1):456-467, 2013. https://doi.org/10.1016/j.jcp.2012.08.026.

[37] R. Ren, H. Li, W. Jiang and M.Y. Song. An efficient Chebyshev-tau method for solving the space fractional diffusion equations. Applied Mathematics and Computation, 224:259-267, 2013. https://doi.org/10.1016/j.amc.2013.08.073.

[38] S.Y. Reutskiy. A new semi-analytical collocation method for solving multi-term fractional partial differential equations with time variable coefficients. Applied Mathematical Modelling, 45:238-254, 2017. https://doi.org/10.1016/j.apm.2016.12.029.

[39] R. Salehi. A meshless point collocation method for 2-D multi-term time fractional diffusion-wave equation. Numerical Algorithms, 74(4):1145-1168, 2017. https://doi.org/10.1007/s11075-016-0190-z.

[40] X. Si, C. Wang, Y. Shen and L. Zheng. Numerical method to initial-boundary value problems for fractional partial differential equations with time-space variable coefficients. Applied Mathematical Modelling, 40(7-8):4397-4411, 2016. https://doi.org/10.1016/j.apm.2015.11.039. 
[41] S. Singh, V.K. Patel and V.K. Singh. Application of wavelet collocation method for hyperbolic partial differential equations via matrices. Applied Mathematics and Computation, 320:407-424, 2018. https://doi.org/10.1016/j.amc.2017.09.043.

[42] M. Uddin and S. Haq. RBFs approximation method for time fractional partial differential equations. Communications in Nonlinear Science and Numerical Simulation, 16(11):4208-4214, 2011. https://doi.org/10.1016/j.cnsns.2011.03.021.

[43] Z. Wang and S. Vong. Compact difference schemes for the modified anomalous fractional sub-diffusion equation and the fractional diffusionwave equation. Journal of Computational Physics, 277:1-15, 2014. https://doi.org/10.1016/j.jcp.2014.08.012.

[44] M. Yaseen, M. Abbas, A.I. Ismail and T. Nazir. A cubic trigonometric B-spline collocation approach for the fractional sub-diffusion equations. Applied Mathematics and Computation, 293:311-319, 2017. https://doi.org/10.1016/j.amc.2016.08.028.

[45] F. Zeng and C. Li. A new Crank-Nicolson finite element method for the timefractional subdiffusion equation. Applied Numerical Mathematics, 121:82-95, 2017. https://doi.org/10.1016/j.apnum.2017.06.011.

[46] F. Zeng, C. Li, F. Liu and I. Turner. Numerical algorithms for time-fractional subdiffusion equation with second-order accuracy. SIAM Journal on Scientific Computing, 37(1):A55-A78, 2015. https://doi.org/10.1137/14096390X.

[47] Y. Zhang, Z. Sun and H. Wu. Error estimates of Crank-Nicolson-type difference schemes for the subdiffusion equation. SIAM Journal on Numerical Analysis, 49(6):2302-2322, 2011. https://doi.org/10.1137/100812707.

[48] F. Zhou and $\mathrm{X}$. Xu. The third kind Chebyshev wavelets collocation method for solving the time-fractional convection diffusion equations with variable coefficients. Applied Mathematics and Computation, 280:11-29, 2016. https://doi.org/10.1016/j.amc.2016.01.029. 